\title{
Efecto de la saturación parcial en la compresibilidad de arenas con rotura de partículas
}

Effect of partial saturation on the compressibility of crushable sands

Fecha de entrega: 14 de diciembre 2018

Fecha de aceptación: 4 de marzo 2019

\section{Rodrigo Osses ${ }^{1,2}$, Carlos Ovalle ${ }^{3}$, Jubert Pineda ${ }^{4}$ y Pedro Barrios ${ }^{5}$}

${ }^{1}$ Departamento Ingeniería de Obras Civiles, Universidad de La Frontera, Francisco Salazar 1145, Temuco, Región de la Araucanía, Chile, rodrigo.osses@ufrontera.cl

2 Departamento de Ingeniería Estructural y Geotécnica, Pontificia Universidad Católica de Chile, Avda. Vicuña Mackenna 4860, Santiago, 7820436, Chile, rosses@uc.cl

${ }^{3}$ Research Institute of Mining and Environment RIME UQAT-Polytechnique, Department of Civil, Geological and Mining Engineering, Polytecnique Montréal, Room A-356, 2900 Édouard Montpetit Blvd, Montreal, QC, H3T 1J4, Canada, carlos.ovalle@polymtl.ca

${ }^{4}$ Priority Research Centre for Geotechnical Science and Engineering, School of Engineering, The University of Newcastle, Callaghan Campus, Building EA, Newcastle, NSW, 2308, Australia, jubert.pineda@newcastle.edu.au

${ }^{5}$ DICTUC S.A., Vicuña Mackenna 4860, Macul, Santiago, 7820436, Chile, pbarrios@dictuc.cl

Se presenta un estudio experimental de laboratorio del efecto de la humedad en la compresibilidad y la fluencia lenta (creep) en dos arenas con rotura de partículas, mediante ensayos edométricos en muestras parcialmente saturadas. La humedad se controla mediante la técnica de control de vapor, de tal manera de cubrir un amplio rango de succión de 0 a $340 \mathrm{MPa}$. Los resultados muestran que, al disminuir la succión (i.e. aumento de humedad), hay más rotura de partículas, la compresibilidad del material aumenta y hay más deformaciones por creep. El estudio de este fenómeno tiene aplicaciones en degradación de materiales granulares ante cambios significativos de humedad, asi como deformaciones por creep en obras geotécnicas, como presas de enrocado, balasto ferroviario, entre otros casos.

Palabras clave: rotura de partículas, creep, arena parcialmente saturada, compresibilidad
An experimental laboratory study of the effect of moisture on compressibility and creep in two crushable sands is presented, by means of oedometric tests in partially saturated samples. The material humidity is controlled by the vapor control technique, in order to cover a wide range of suction from 0 to $340 \mathrm{MPa}$. The results show that, as the suction decreases (i.e. humidity increases), both compressibility and creep deformations increase, due to the higher amount of particle breakage. This phenomenon has applications in degradation of granular materials under significant changes of humidity, as well as deformations by creep in geotechnical works, such as rockfill dams, railway ballast, among other cases.

Keywords: particle breakage, creep, unsaturated sand, compressibility

\section{Introducción}

Diversos estudios han demostrado que el principal mecanismo responsable de la compresibilidad no lineal de materiales granulares es la rotura de partículas (Vesic y Clough, 1968; Lade et al., 1996; Biarez y Hicher,1997; Dano et al., 2017; Ovalle, 2013). Además, es sabido que el aumento de humedad del material incrementa la magnitud de roturas y, en consecuencia, la compresibilidad (Oldecop y Alonso, 2003; Ovalle, 2018). La explicación estaría dada por el fenómeno conocido en la literatura técnica como Stress Corrosion Cracking SCC, el cual establece que, en un material bajo tensión, parte de las microfisuras existentes se exponen, facilitando el ingreso de la humedad. Este proceso genera corrosión y una disminución de la resistencia al fracturamiento de las partículas de arena y agregados de roca (Atkinson, 1982). Así, al incrementarse la tensión y/o la humedad, un material granular experimenta más degradación por rotura de partículas. Además, el SCC puede ser un proceso lento y generar fluencia lenta (creep) por rotura de partículas desfasada (Oldecop y Alonso, 2007; Ovalle et al., 2015). En obras geotécnicas, algunas aplicaciones para el estudio de este fenómeno lo constituyen, por ejemplo, los asentamientos en el tiempo 
en grandes presas de material granular, o en pilotes en arena. Este fenómeno ha sido poco estudiado y existe poca información reportada acerca de la influencia de la humedad en la compresibilidad de materiales granulares.

En este trabajo se presenta un estudio experimental de laboratorio para evaluar la compresibilidad y el creep en materiales granulares con rotura de partículas. Se seleccionaron dos arenas de diferente origen y composición mineralógica, las que se ensayaron en compresión edométrica con niveles de tensión de hasta 1.6 MPa, a razón de un incremento de carga por día y en condición parcialmente saturada. La humedad de las muestras se controló mediante la técnica de control de vapor en una celda de compresión edométrica.

\section{Materiales ensayados}

Dos muestras de diferente origen y composición mineralógica fueron seleccionadas:

- Colina: roca chancada de cantera de la comuna de Colina en la Región Metropolitana, Chile, extraída desde una pequeña cantera artesanal. El material corresponde a una roca intrusiva perteneciente al Grupo Volcánico de la era del Oligo-Mioceno y llamada Andesita (Vergara y Drake, 1979), principalmente compuesta por cuarzo.

- Pilbara: roca chancada proveniente de la región de Pilbara en Western Australia, Australia, obtenida de la roca estéril de una mina de hierro. Es un material de partículas erodadas y transportadas (coluvión) derivadas de una Formación de Bandas de Hierro de la era Precámbrica (Linero et al., 2017), y está principalmente compuesta por $\mathrm{Fe}$.

Fotos de los granos de las muestras se presentan en la Figura 1 y las curvas granulométricas de cada material se muestran en la Figura 2.

a)

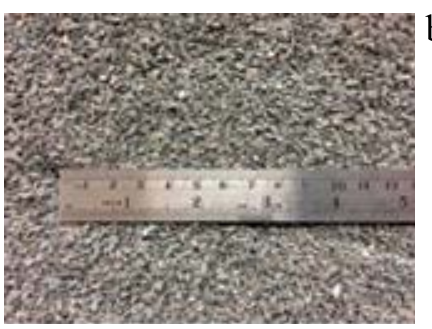

Figura 1: Fotos de las muestras estudiadas de: a) Colina (Sample 1) y b) Pilbara (Sample 2)

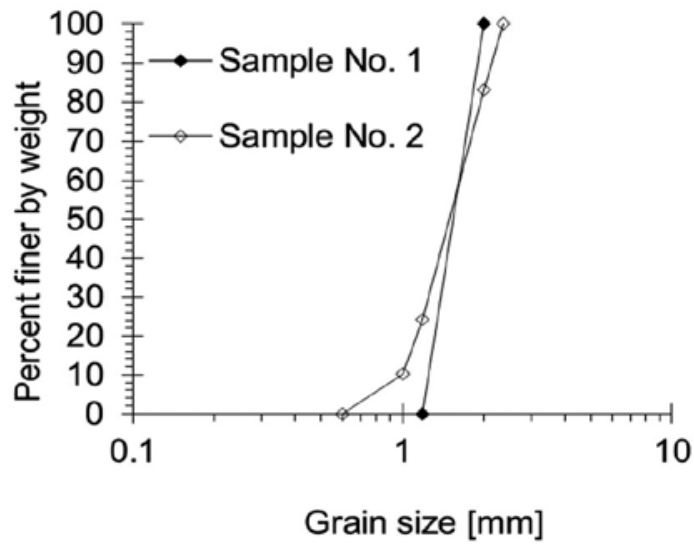

Figura 2: Distribuciones granulométricas de las arenas ensayadas de Colina y Pilbara

\section{Metodología de ensayos}

Se realizaron ensayos de compresión edométrica con carga constante en muestras parcialmente saturadas a distintas humedades. Los siguientes niveles de tensión vertical fueron aplicados en cada uno de los ensayos: 10, 25, 49, $98,196,392,785$ y $1569 \mathrm{kPa}$, a través de un incremento de carga por día. Algunos ensayos fueron inundados con agua desmineralizada 24 horas después de la tensión máxima, con el objetivo de evaluar el fenómeno de colapso y creep. Durante todos los niveles de esfuerzo aplicados la deformación fue medida y registrada a partir de $0.1 \mathrm{~min}$ y hasta 24 horas (1440 min).

La saturación parcial se impuso mediante la técnica de control de vapor, a través de un circuito de aire cerrado que integra la muestra en un ensayo edométrico. La humedad relativa es controlada dentro del circuito y, una vez alcanzado el equilibrio, se puede mantener constante la humedad y por lo tanto la succión en un material parcialmente saturado (Fredlund y Rahardjo, 1993). La succión puede obtenerse mediante la Ley de Kelvin conociendo la temperatura $T$ y la humedad relativa $R H$ del aire en los poros:

$$
\psi=-\frac{R T}{v_{w 0} \omega_{v}} \ln (R H)
$$

donde $\psi$ es la succión total, $R$ es la constante universal de los gases $(8.31432 \mathrm{~J} /[\mathrm{mol} \mathrm{K}]), v_{w 0}$ es el volumen específico del agua, o el inverso de la densidad del agua $\left(1 / \rho_{\mathrm{w}} \mathrm{m}^{3} / \mathrm{kg}\right)$ y $\omega_{v}$ es la masa molecular del vapor de agua $(18.016 \mathrm{~kg} / \mathrm{mol})$.

El circuito cerrado de vapor consiste en una bomba de 
,

.

aire que genera un flujo circulante entre la muestra y una solución salina saturada, según se muestra en la Figura 3. El sistema incluye un control de presión para no afectar las tensiones totales en el ensayo (presión de aire $<2$ $\mathrm{kPa}$ ), además de 2 higrómetros para el monitoreo de la temperatura y humedad relativa del aire antes y después de pasar a través de la muestra de arena. La Tabla 1 muestra los valores medidos y la succión calculada mediante la ecuación (1). En la Figura 4 se presenta la variación en el tiempo de la humedad y succión para los ensayos en la arena de Colina.

a)

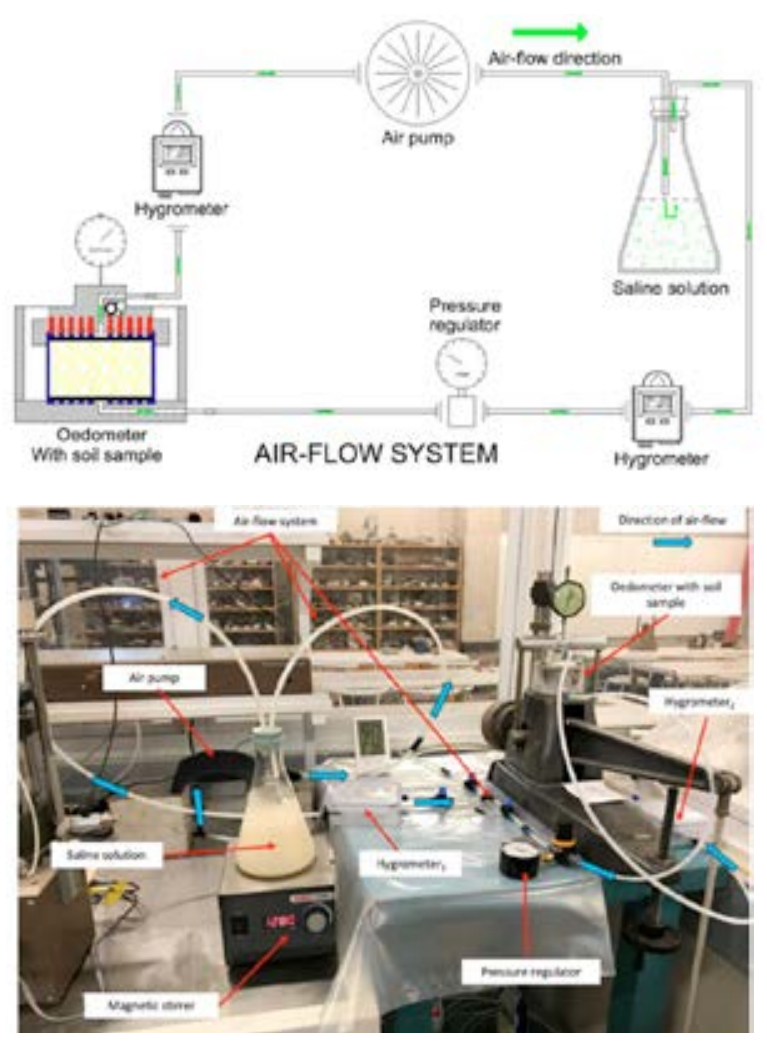

Figura 3: Técnica de control de vapor: a) esquema del circuito hermético y b) circuito real

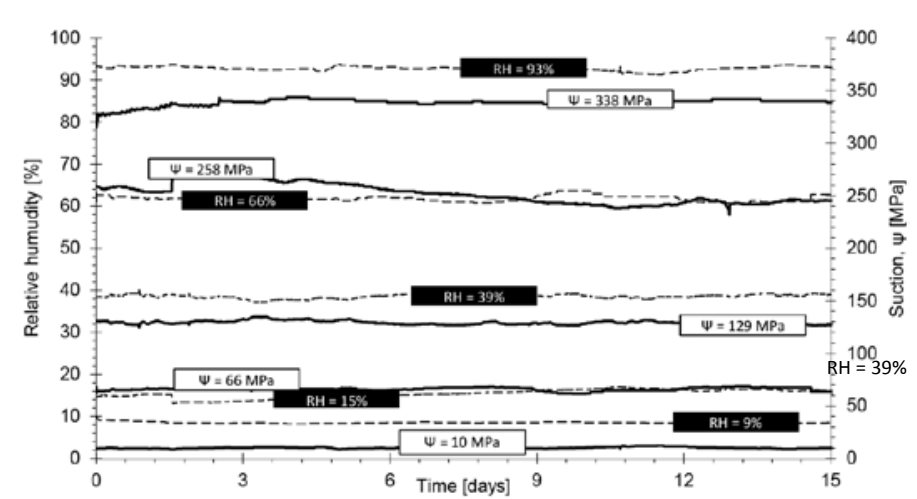

Figura 4: Humedad relativa $R H$ y $\psi$ succión total medidas en muestras de Colina
Tabla 1: Temperatura, humedad relativa y succión en cada ensayo.

\begin{tabular}{|c|c|c|c|c|}
\hline Muestra & $\begin{array}{c}\text { Solución } \\
\text { salina }\end{array}$ & $\begin{array}{c}T \\
\text { promedio, } \\
{ }^{\circ} \mathrm{C}\end{array}$ & $\begin{array}{c}\text { RH pro- } \\
\text { medio, } \%\end{array}$ & $\begin{array}{c}\text { Succión } \\
\text { total, } \\
\text { MPa }\end{array}$ \\
\hline Colina & $\mathrm{NaOH}$ & 18.0 & 9 & 338 \\
\hline Colina & $\mathrm{KOH}$ & 26.5 & 15 & 258 \\
\hline Colina & $\mathrm{MgCl}_{2}$ & 20.9 & 39 & 129 \\
\hline Colina & $\mathrm{NaOH}$ & 24.9 & 66 & 66 \\
\hline Colina* & $\mathrm{NaBr}^{2}$ & 24.9 & 66 & 66 \\
\hline Colina & $\mathrm{K}_{2} \mathrm{SO}_{4}$ & 20.5 & 93 & 10 \\
\hline Colina & $\begin{array}{c}\mathrm{H}_{2} \mathrm{O} \mathrm{desmi-}_{\text {neralizada }} \\
\text { nilbara }\end{array}$ & - & - & 0 \\
\hline NaOH & 23.9 & 9 & 324 \\
\hline Pilbara & $\mathrm{MgCl}_{2}$ & 25.5 & 45 & 109 \\
\hline Pilbara & $\begin{array}{c}\mathrm{H}_{2} \mathrm{O} \text { desmi- } \\
\text { neralizada }\end{array}$ & - & - & 0 \\
\hline
\end{tabular}

La variación de humedad de la arena en el sistema de control de vapor puede estimarse a partir de las curvas de retención de humedad. Por ejemplo, la Figura 5 muestra estas curvas para la arena de Pilbara, obtenidas en distintas fracciones de tamaño mediante el método del espejo refrigerado con un equipo WP4C (determinación del punto de rocío). El proceso se inicia con un humedecimiento progresivo de las muestras (datos en color negro), hasta alcanzar el mínimo valor de succión y por consiguiente la máxima humedad (datos en color azul) y finalmente un proceso de secado progresivo (datos en color rojo). Los resultados muestran que en los rangos de succión impuestos en el sistema de control de vapor el material de Pilbara presenta una humedad de hasta $2.5 \%$.

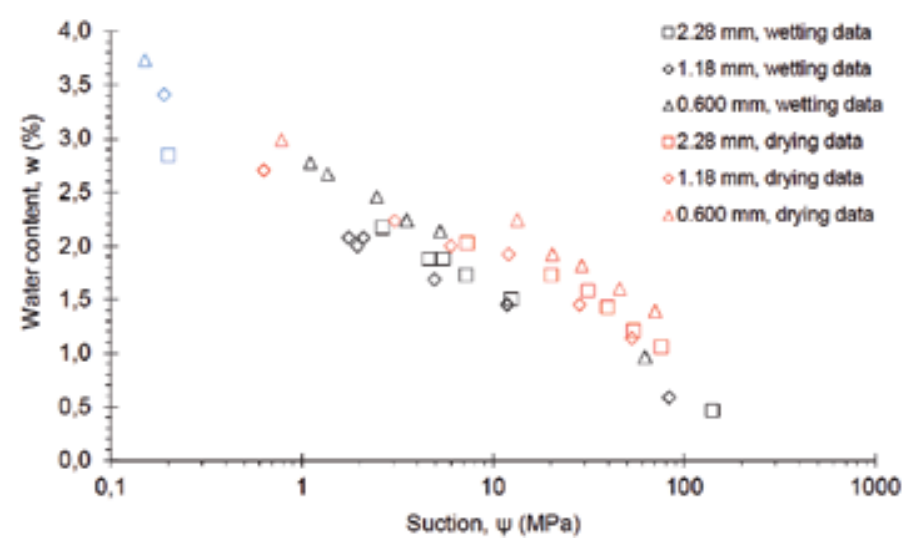

Figura 5: Curvas de retención de humedad Pilbara 


\section{Resultados y análisis}

La Figura 6 presenta las curvas de comprensión edométrica obtenidas para cada muestra y para cada succión aplicada. Las muestras inundadas a la tensión máxima corresponden a los valores de succión 10 y $66 \mathrm{MPa}$ para Colina y 109 MPa para Pilbara. Para ambos casos, las muestras saturadas alcanzan los mayores valores de deformación: $8.2 \%$ y $3.8 \%$ en Colina y Pilbara, respectivamente; mientras que para valores de succión mayores a $300 \mathrm{MPa}$ las deformaciones son de $4.6 \%$ y $3.1 \%$ para Colina y Pilbara respectivamente.

a)
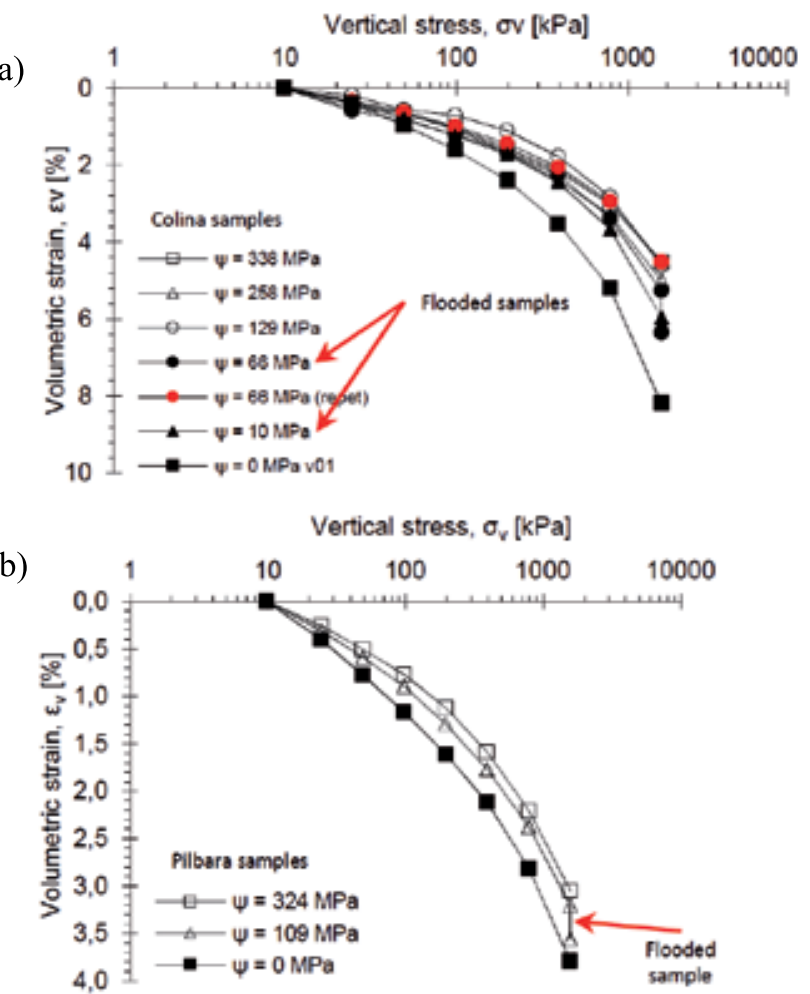

Figura 6: Curvas de compresión edométrica para las muestras de: a) Colina y b) Pilbara

LaFigura 7 presenta las curvas de evolución de la granulometría al final de cada ensayo, donde se incluye la granulometría inicial seleccionada para cada muestra de suelo.

La Figura 8 muestra un ejemplo de las curvas de deformación en el tiempo obtenidas para la muestra de Colina con $\psi=334 \mathrm{MPa}$.

Como se esquematiza en la Figura 9, la compresibilidad puede caracterizarse mediante el parámetro $C_{\mathrm{c}}=\Delta e /$ $\Delta \log \left(\sigma_{\mathrm{v}}\right)$, mientras que la compresibilidad secundaria, o creep, mediante $C_{\alpha}=\Delta e / \Delta \log (t)$ (Mesri y Vardhanabhuti, 2009). Las Figuras 10 y 11 muestran los coeficientes $C_{\mathrm{c}}$ y $C_{\alpha}$ calculados para cada incremento de tensión en todos los ensayos. Los resultados muestran que la compresibilidad y las deformaciones por creep aumentan con la tensión aplicada y con la humedad.

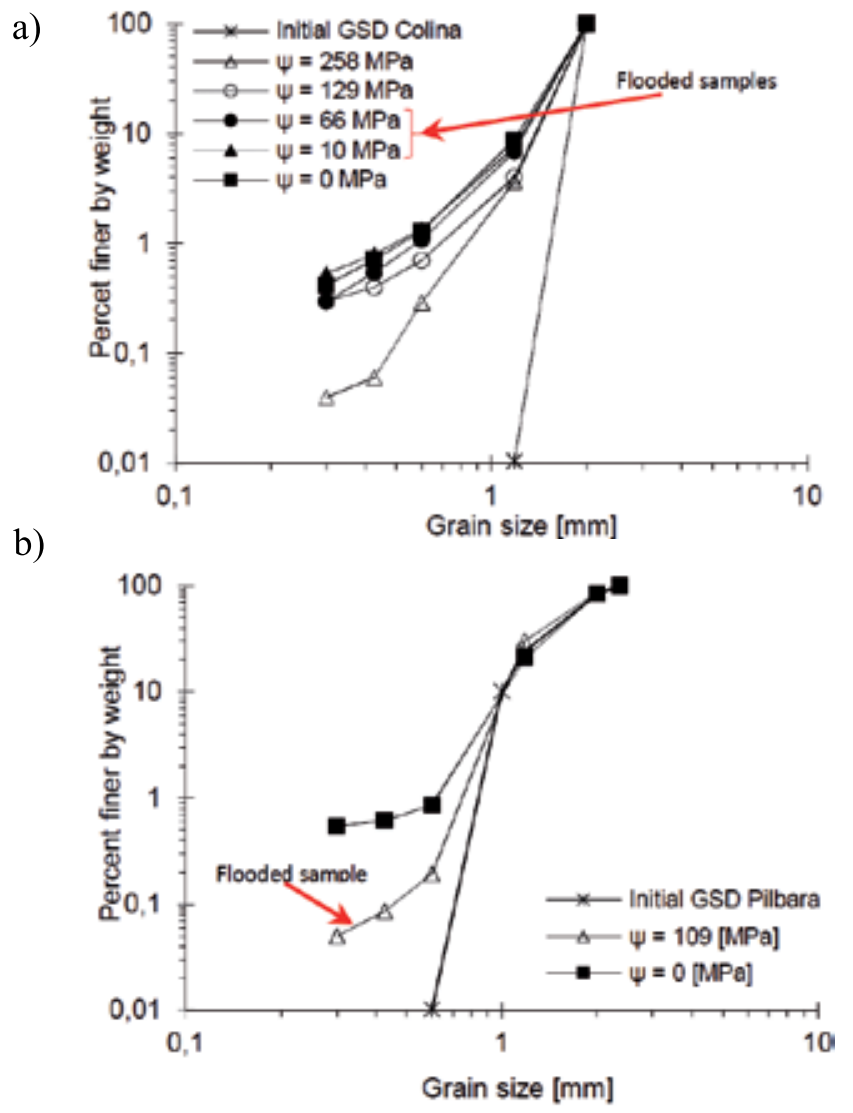

Figura 7: Granulometrías de las muestras de: a) Colina y b) Pilbara

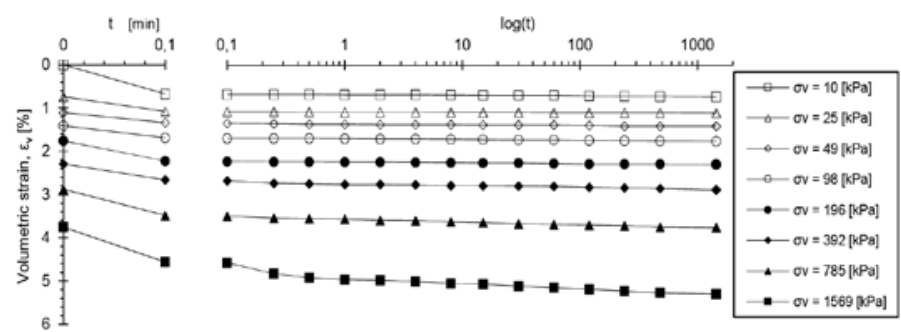

Figura 8: Curvas de deformación en el tiempo en arena de Colina $\operatorname{con} \psi=334 \mathrm{MPa}$

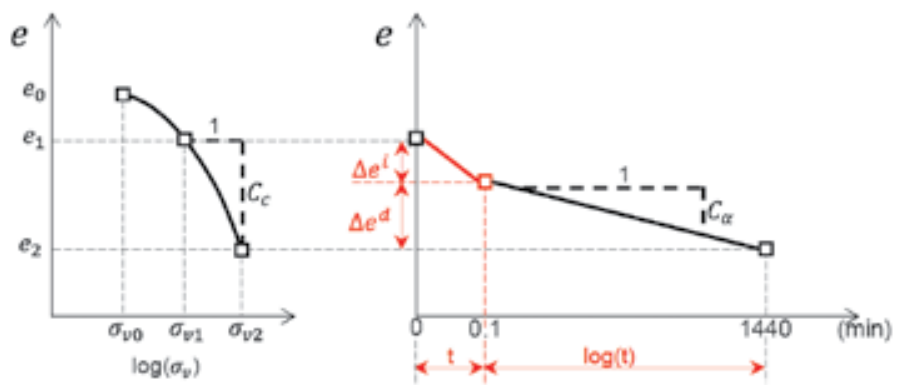

Figura 9: Esquema de cálculo de los coeficientes: a) $\mathrm{C}_{\mathrm{c}}$ y b) $\mathrm{C}_{\alpha}$ 
a)

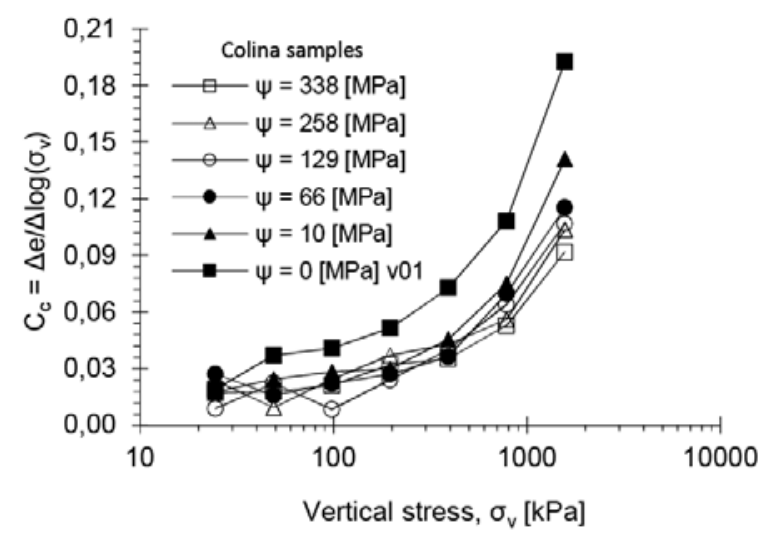

b)

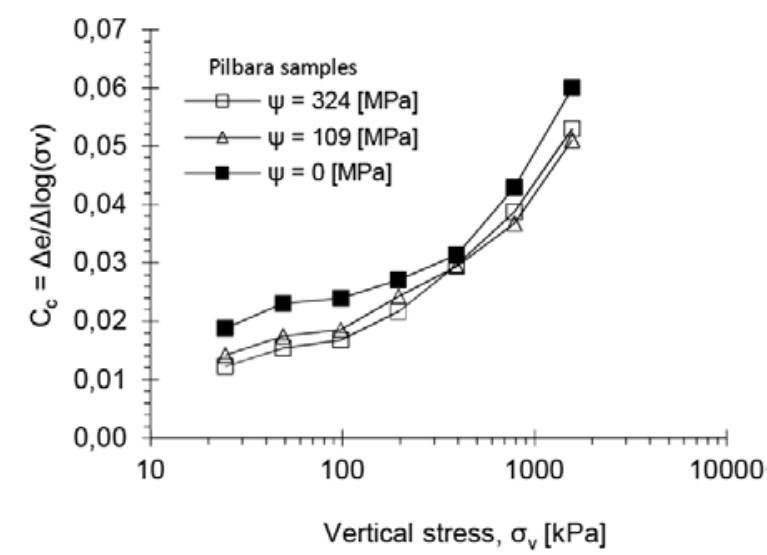

Figura 10: Variación de $\mathrm{C}_{\mathrm{c}}=\Delta \mathrm{e} / \Delta \log \left(\sigma_{\mathrm{v}}\right)$ versus la tensión vertical para las muestras de: a) Colina y b) Pilbara

a)

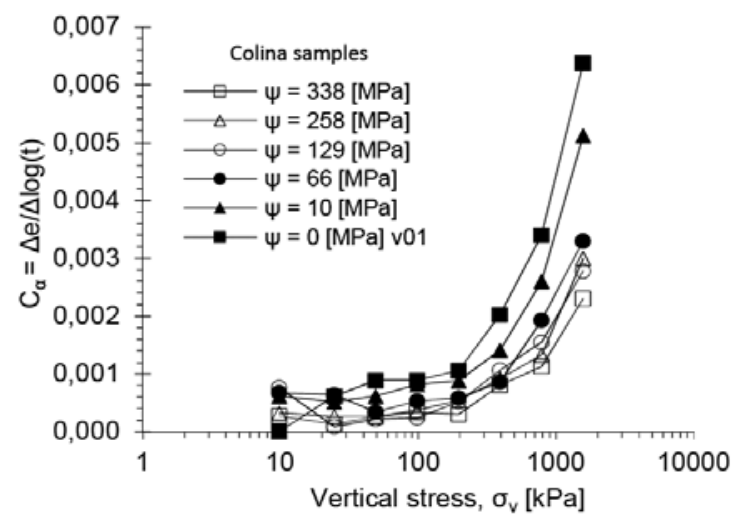

b)

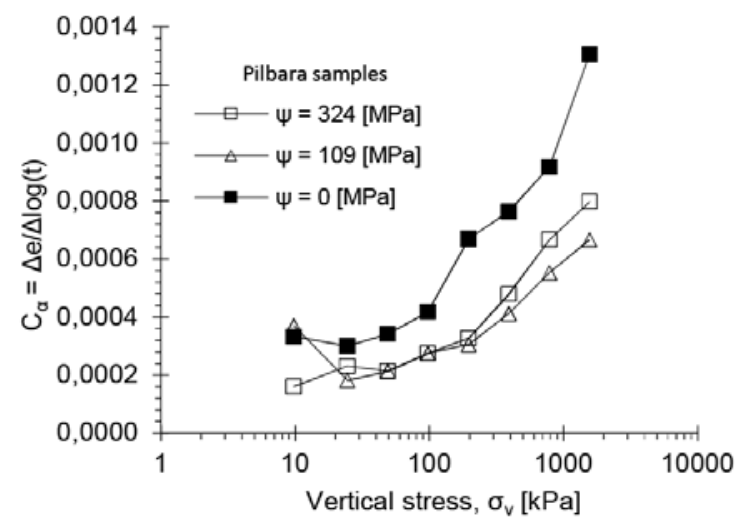

Figura 11: Variación de $\mathrm{C}_{\alpha}=\Delta \mathrm{e} / \Delta \log (\mathrm{t})$ versus la tensión vertical para las muestras de: a) Colina y b) Pilbara
Según la Figura 8 y el esquema de la Figura 9, en cada incremento de tensión se generan 2 mecanismos de deformación: instantáneo y desfasado (creep). Así, la deformación puede desacoplarse en instantánea $\varepsilon^{i}$ (medida a 0.1 min en este estudio) o de creep $\varepsilon^{d}$ (medida a 1440 min en este estudio). Basados en este concepto, Oldecop y Alonso (2001) propusieron un modelo constitutivo para materiales granulares incluyendo el efecto de creep debido a la rotura de partículas, donde el índice de compresibilidad en el tiempo $\lambda^{d}$ depende de la succión, mientras que el índice de compresibilidad instantáneo $\lambda^{i}$ es independiente de la succión.

$$
d \varepsilon=d \varepsilon^{i}+d \varepsilon^{d}=\lambda^{i} d \sigma+\lambda^{d}(\psi) d \sigma
$$

Las Figuras 12 y 13 muestran los valores de $\varepsilon^{i}$ y $\varepsilon^{d}$, respectivamente, para las arenas de Colina y Pilbara. En concordancia con la hipótesis de Oldecop y Alonso (2001), en ambos materiales se obtiene una relación $\varepsilon^{d}$ versus $\sigma_{v}$ que depende de la succión. Sin embargo, en la arena de Colina se observa que la relación $\varepsilon_{i}$ vs $\sigma_{v}$ también depende de la succión, i.e. $\lambda^{i}=\lambda^{i}(\psi)$.

a)

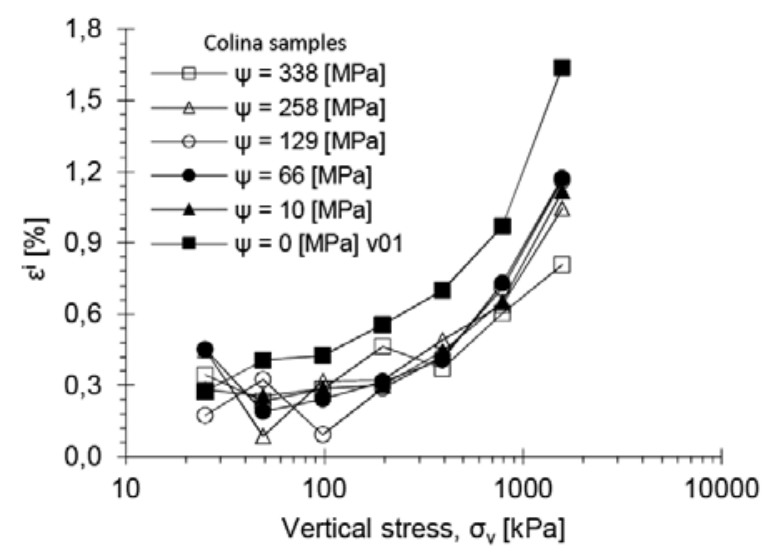

b)

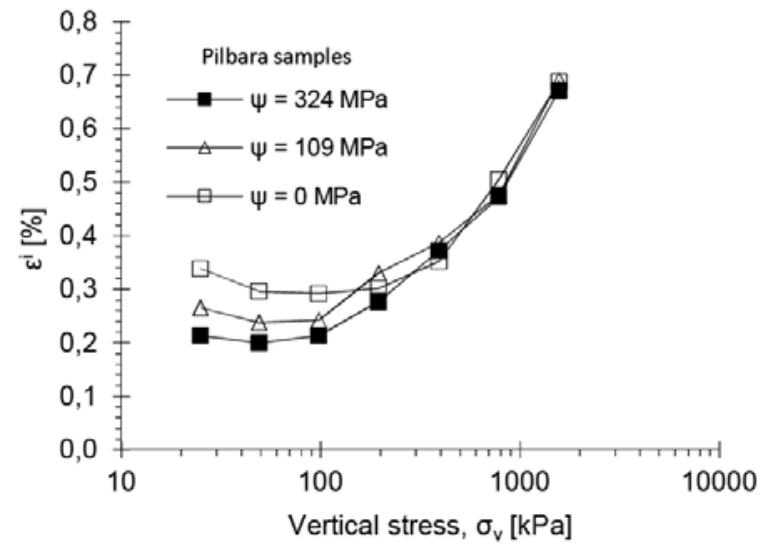

Figura 12: Deformación instantánea $\varepsilon^{i}$ para las muestras de: a) Colina y b) Pilbara 
a)

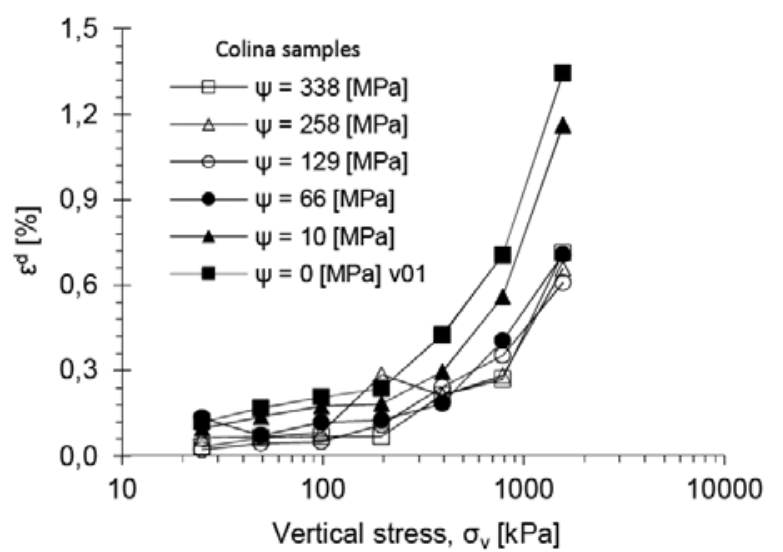

b)

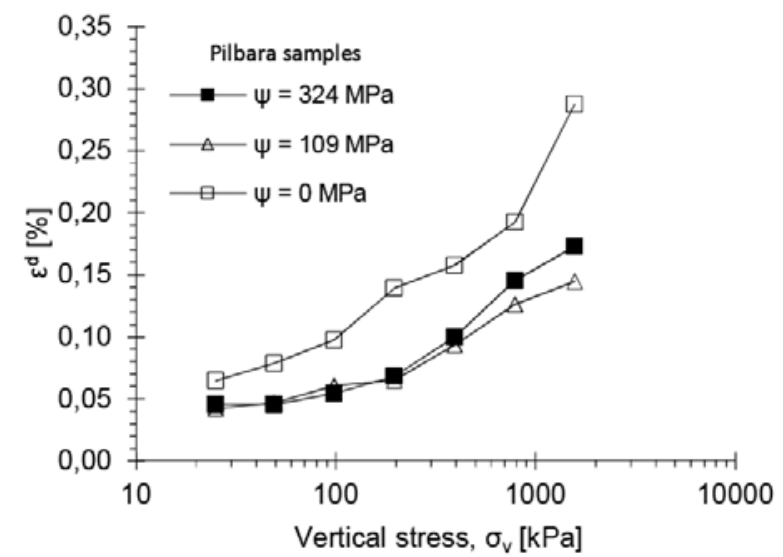

Figura 13: Deformación desfasada o de creep $\varepsilon^{d}$ para las muestras de: a) Colina y b) Pilbara

\section{Conclusiones}

Se presenta un estudio experimental de laboratorio del efecto de la humedad en la compresibilidad y la fluencia lenta (creep) de dos arenas con rotura de partículas, mediante ensayos edométricos en muestras parcialmente saturadas. Las principales conclusiones del estudio son las siguientes:

- La compresibilidad aumenta con la humedad, debido a un incremento de la rotura de partículas.

- Los ensayos muestran que se generan deformaciones por creep, con un índice de compresibilidad secundario $\left(\mathrm{C}_{\alpha}\right)$ que aumenta con la disminución de la succión, es decir, a mayor humedad hay más deformaciones por creep.

- El mecanismo de deformación en un incremento de tensión se puede desacoplar en una deformación instantánea y una desfasada (creep); para un nivel de tensión dado, ambos valores dependen de la succión.

\section{Agradecimientos}

Este estudio se enmarca en el proyecto FONDECYT 11150084, financiado por CONICYT. El primer autor agradece el financiamiento de la Beca de Doctorado en Chile otorgada por CONICYT.

\section{Referencias}

Atkinson, B. (1982). Subcritical crack propagation in rocks: experimental results and applications. Journal of Structural Geology 4(1), 41-56

Biarez, J. et Hicher P.Y. (1997). Influence de la granulométrie et de son évolution par ruptures de grains sur le comportement mécanique de matériaux granulaires. Revue Française de Génie Civil 1(4), 607-631

Dano, C., Ovalle, C., Yin, Z., Daouadji, A. and Hicher, P.Y. (2017). Behavior of granular materials affected by grain breakage. Advances in Multi-Physics and Multi-Scale Couplings in Geo-Environmental Mechanics, 1st Edition. Eds. Nicot, F. and Millet, ISTE Press, 95-132

Fredlund, D.G. and Rahardjo, H. (1993). Soil mechanics for unsaturated soils. John Wiley \& Sons Inc.

Lade, P., Yamamuro, J. and Bopp, P. (1996). Significance of particle crushing in granular materials. Journal of Geotechnical Engineering 122(4), 309-316

Linero, S., Fityus, S., Simmons, J., Lizcano, A. and Cassidy J. (2017). Trends in the evolution of particle morphology with size in colluvial deposits overlying channel iron deposits. Powders and Grains 2017 - $8^{\text {th }}$ International Conference on Micromechanics on Granular Media, EPJ Web of Conferences 140, 14005. EDP Sciences

Mesri, G. and Vardhanabhuti, B. (2009). Secondary compression. Canadian Geotechnical Journal 46(4), 369-392

Oldecop, L.A. and Alonso, E.E. (2007). Theoretical investigation of the time-dependent behaviour of rockfill. Géotechnique 57(3), 289-301

Oldecop, L.A. and Alonso E.E. (2003). Suction effects on rockfill compressibility. Géotechnique 53(2), 289-292

Oldecop, L.A. and Alonso E.E. (2001). A model for rockfill compressibility. Géotechnique 51(2), 127-139 
Ovalle, C. (2018). Role of particle breakage in primary and secondary compression of wet and dry sand. Géotechnique Letters 8(2), 161-164

Ovalle (2013). Contribution à l'étude de la rupture des grains dans les matériaux granulaires. Doctoral thesis, Ecole Centrale de Nantes, France.

Ovalle, O., Dano, C., Hicher, P.Y. and Cisternas, M. (2015). Experimental framework for evaluating the mechanical behavior of dry and wet crushable granular materials based on the particle breakage ratio. Canadian Geotechnical Journal 52(5), 587-598
Vergara, M. y Drake, R. (1979). Edades K/Ar en secuencias volcánicas continentales postneocomianas de Chile Central: su depositación en cuencas intermontanas restringidas. Revista de la Asociación Geológica Argentina 34, 42-52

Vesic, A. and Clough, G. (1968). Behavior of granular materials under high stresses. Journal of the Soil Mechanics and Foundations Division 94(3), 661-688 